\title{
Utility of Exfoliative Cytology of Voided Urine in Comparison with Ultrasonogram and Cystoscopy Findings in the Diagnosis of Urothelial Cancers
}

\author{
SM Badruddoza ${ }^{1}$, FA Azim ${ }^{2}$, AJE Nahar Rahman ${ }^{2}$, M. Kamal ${ }^{2}$

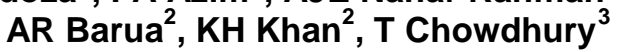

\begin{abstract}
Carcinoma of the urinary bladder affects men more often than women and occurs in patients over the age of 50 years. Transitional cell carcinoma (TCC) comprises about $90 \%$ of all primary tumor of urinary bladder. In this study a total of 57 cases were taken. The aim of the study was to establish diagnostic role of voided urine cytology, ultrasonography and cystoscopy in urothelial cancers. Out of 57 cases, cytological diagnosis was positive in 53 cases (93\%) and negative in 4 cases (7\%). There were no atypical or suspicious cases. Of the total cases, cytology was negative only in 7\% cases. Among 42 invasive carcinomas 14 (33\%) grade III invasive flat carcinoma, one (2\%) invasive papillary adenocarcinoma and two (5\%) invasive squamous cell carcinoma, each of them gave $100 \%$ positive cytology. Of the remaining 25 (60\%) invasive papillary TCC, 21 were in grade II and 4 were in grade III. They gave $90 \%$ and $100 \%$ positive cytologic results. Ultrasonogram of urinary bladder gave $95 \%$ (approx.) positive results and out of 57 cases, 3 cases failed to give positive results in ultrasonogram (USG) of the urinary tract. Cystoscopy detected tumor mass in $98 \%$ cases and it gave false negative result in $2 \%$ cases. Fifty-two tumors had a diameter ranging $1.1-6 \mathrm{~cm}$. Single lesions were seen in 40 cases, double lesion in one case and multiple in 13 cases. The probability of later invasive carcinomas nearly tripled in-patients with multiple lesions on presentation (13.6\%) compared with those who presented with a single lesion (4.6\%). Consistent with previously published data, this study showed the highest diagnostic accuracy with high-grade tumors and lowest with low-grade tumors. So in a proper clinical setting, non-invasive technique like voided urine cytology and ultrasonography could be done as a first line cost effective method in the diagnosis of urothelial cancers.
\end{abstract}

Key words: Exfoliative cytology, voided urine, cystoscopy, urothelial cancer

TAJ 2017; 30: No-2: 39-46

\section{Introduction:}

Carcinoma of the urinary bladder affects men more often than women at a ratio of $3: 1$ to $4: 1{ }^{1}$ Most cases present in-patients over the age of 50 years, but they can also occur in younger adults and children. Transitional cell carcinoma (TCC) comprises about $90 \%$ of all primary tumor of urinary bladder. ${ }^{2}$

Haematuria is the most common symptom of transitional cell carcinoma, followed by symptoms related to associated urinary tract infection. Dysuria is more often seen with high-grade tumors

1 Professor, Department of Pathology, Rajshahi Medical College, Rajshahi.

2 Professor, Department of Pathology, BSMMU, Dhaka.

${ }^{3}$ Professor, Department of Pathology, BSMMU, Dhaka. 
perhaps as a result of involvement of the bladder wall. $^{2}$

The urothelial tumors may be papillary or nonpapillary (flat) and invasive or in-situ (noninvasive). ${ }^{3,4}$ Transitional cell carcinoma of the urinary bladder is generally divided into three groups: superficial papillary, carcinoma in-situ and invasive. Superficial bladder tumors are largely grade-I or II papillary transitional cell carcinomas that may or may not invade the lamina propria. ${ }^{5}$ Usually, non-invasive lesions are often referred to as "superficial" to differentiate them from carcinoma in-situ (C.I.S). ${ }^{4}$

It has now been documented that in about $80 \%$ of the cases, primary invasive carcinoma of the bladder are not preceded by papillary tumors, hence the conclusion that most of these tumors are derived from invisible and asymptomatic flat lesions namely carcinoma in-situ. ${ }^{6,7,8}$

The principal features of carcinoma in-situ of the urinary bladder are that cystoscopically it may mimic inflammation or there may be no cystoscopic or sonographic abnormality whatsoever. The most important property of flat carcinoma in-situ is its ability to progress to invasive carcinoma. If untreated, carcinoma insitu will progress to invasive carcinoma in at least $60 \%$ of all patients within 5 years. Because the lesions produces only non-specific symptoms or may be asymptomatic, its diagnosis is based either on cytology of voided urine or on incidental biopsies of bladder epithelium. ${ }^{6}$

One of the major accomplishments of urine cytology is the detection of urothelial carcinoma in its preclinical phase; long before its cytoscopic and radiographic recognition. ${ }^{15}$ Cytological examination of voided urine is the technique of choice for detection, diagnosis and follow up of tumors of the lower urinary tract. The most important value of cytology of voided urine is the diagnosis of clinically unsuspected case of carcinoma particularly carcinoma in-situ. ${ }^{9}$

Moreover cytological examination of voided urine is the only totally harmless non-invasive method, ${ }^{9,10}$ easy to obtain ${ }^{10}$ and can be done on an outpatient basis. ${ }^{11}$ Cytology has the distinct advantage of sampling of entire urothelial mucosa. $^{3}$ The simplicity, convenience and accuracy of multiple voided urine cytology justifies its continued use as a first line diagnostic and detection technique. ${ }^{10} \mathrm{~A}$ definitive diagnosis of urinary bladder tumor cannot, of course, be made without cystoscopy and biopsy but voided urine cytology \& ultrasonography along with its different modification assists the physician to predict the presence of bladder tumor and plan the treatment that may be necessary at cystoscopy.

In summary, it may be concluded that urinary cytology can be a useful diagnostic adjunct in the study of patients with transitional cell carcinoma. ${ }^{12}$ and in the clinical setting, urine cytology, USG and cystoscopy complement each other as diagnostic tools, adding greatly to the clinician's ability to detect occult and particularly high grade bladder tumors. ${ }^{13}$

\section{Aims and Objectives:}

This study is designed to see correlation of voided urine cytology with ultrasonogram and cystoscopy findings and relative frequencies of different malignant tumors of urinary bladder.

\section{Materials and Methods:}

The study was carried out in the Department of Pathology, B.S.M.M.U. Dhaka, during one year period.

A. Selection of the Patients: A total of fifty seven patients clinically suspicious of having urinary bladder malignancy or histologically confirmed patients, admitted in the Urology Department of B.S.M.M.U., D.M.C.H. and private clinics were included in this study.

\section{B. Cytological examinations:}

Selection of Specimens: From all patients at each admission, the diagnostic work up included cytological examinations of three samples of morning's second voided urine. ${ }^{3}$

Sample Preparations: Urine samples should be sent to the laboratory within three hours of collection. Fifty (50) $\mathrm{ml}$ of urine specimen is centrifuged at $10 \mathrm{~g}$ for 5-10 minutes, the supernatant decanted and the pellet is directly smeared on glass slides. ${ }^{10}$ Albuminized slides are 
recommended for better attachment of cells to the slides. ${ }^{3,6,14-16}$

Pattern of Smears (Smear Criteria): The smears undergoing cytological examination were diagnosed as:

\section{Negative}

2. Atypia or Atypical

3. Suspicious

4. Positive or malignant cells present

5. Non diagnostic or unsatisfactory

Finally the negative and atypical diagnoses were combined in to one group (cytologic negatives) and the suspicious and positive diagnoses were combined in to a second group (cytologic positives). ${ }^{9,12,17-20}$

\section{Ultrasound examination of kidney, ureter} and urinary bladder region: Per abdominal sonographic scan of KUB region, especially lower abdomen was done in full bladder state in supine position.

The sonographic variables are:

1. Size of the tumor

2. Number of the tumor

3. Peri-vesical extension

4. Lymphadenophaty

D. Cystoscopic examination of the urinary bladder:

1. Cystoscopy done under spinal anaesthesia followed by biopsy and histopathological Examination. Tissue diagnosis was compared with the cytological diagnosis in this study. Cytologic diagnosis was constantly earlier than pathologic diagnosis in this series. Biopsy / biopsies were taken usually from the lesion proper and represent cystoscopic biopsy, cystectomy or nephrectomy specimens. All biopsy specimens either cystoscopic biopsy or cystectomy specimens were collected in $10 \%$ formalin. After careful gross examination, the specimens were subjected to routine processing. The Haematoxylin and Eosin stained slides were critically examined to evaluate histological features.

2. All necessary and relevant information regarding patients were recorded methodically and meticulously as far as possible. All data were evaluated by standard statistical methods.

\section{Results and Observations:}

The purpose of the present study was to establish diagnostic usefulness of voided urine cytology, ultrasonography and cystoscopy in the evaluation of urinary bladder tumor.

A total of 57 cases were collected from the Urology Departments of the B.S.M.M.U., D.M.C.H. and private clinics during one year period. Out of total 57 cases, 55 were from the urinary bladder and the rest two was from transitional cell carcinoma of the renal pelvis.

\section{Cytologic diagnoses in $\mathbf{5 7}$ cases:}

There were no atypical, suspicious or unsatisfactory cases. Out of 57, 53 (92.99\%) were given positive for malignancy and 4 (7.01\%) were negative.

Table I: Cytologic diagnosis of 57 cases.

\begin{tabular}{llccc}
\hline No. & & Cytologic diagnosis & No. of cases & Percentage \\
\hline 1. & Negative & & 04 & $7.01 \%$ \\
2. & Atypical & & 00 & $00 \%$ \\
3. & Suspicious & & 00 & $00 \%$ \\
4. & Positive & & 53 & $92.99 \%$ \\
5. & Unsatisfactory & \multirow{2}{*}{ Total } & 00 & $00 \%$ \\
& & & 57 & $100 \%$ \\
\hline
\end{tabular}




\section{Correlation of number of tumors and results of cytology:}

Although not the number but the type of histologic abnormality of the tumor or associated epithelium influence the result of cytology, still when the number of tumors are high, the type of associated high grade changes in the tumor or associated epithelium is likely to be high and so the result of cytology. In table II, it was observed that in two or multiple tumors the result was $100 \%$ and in case of single tumor, it was $90 \%$.

Table II: Correlation between number of tumors at cystoscopy and result of cytology.

\begin{tabular}{ccccccc}
\hline No. of Tumors & No. & \multicolumn{3}{c}{ Result of cytology } \\
& & \multicolumn{2}{c}{ Positive } & \multicolumn{2}{c}{ Negative } \\
& & No. & \% & No. & \% \\
\hline One & 40 & 36 & $90 \%$ & 04 & $10 \%$ \\
Two & 01 & 01 & $100 \%$ & 00 & $00 \%$ \\
Multiple & 13 & 13 & $100 \%$ & 00 & $00 \%$ \\
Total* & 54 & 50 & $92.60 \%$ & 04 & $7.40 \%$ \\
\hline
\end{tabular}

* Is situ Carcinoma: Detected during Cystoscopy as oedematous congested mucosa.

* Two renal pelvis mass: Cystoscopy was not done.

Tumor configuration seen at cystoscopy and correlation with results of cytology:

In table III, cytologic findings are correlated with configuration of the tumor as viewed by cystoscopist. Among the sessile tumors, the cytologic result was $100 \%$ positive. However among the largest group of 38 papillary tumors, only $89.48 \%$ were positive. In a single case of carcinoma in situ though failed to diagnose during cystoscopy, the result of voided cytology was $100 \%$.

Table III: Tumors seen at cystoscopy: configuration and result of cytology.

\begin{tabular}{|c|c|c|c|c|c|}
\hline \multirow[t]{3}{*}{ Tumor configuration } & \multirow[t]{3}{*}{ No. } & \multicolumn{4}{|c|}{ Result of cytology } \\
\hline & & \multicolumn{2}{|c|}{ Positive } & \multicolumn{2}{|c|}{ Negative } \\
\hline & & No. & $\%$ & No. & $\%$ \\
\hline In-situ * & 01 & 01 & $100 \%$ & 00 & 00 \\
\hline Papillary & 38 & 34 & $89.48 \%$ & 04 & $10.52 \%$ \\
\hline Sessile & 16 & 16 & $100 \%$ & 00 & $00 \%$ \\
\hline Total $^{\Psi}$ & 55 & 51 & $92.73 \%$ & 04 & $7.27 \%$ \\
\hline
\end{tabular}

* In-situ Carcinoma: Detected during Cystoscopy as oedematous congested mucosa.

${ }^{\Psi}$ Two renal pelvis mass: Cystoscopy was not done.

Ultrasonography versus Cystoscopy of the urothelial tumors:

Table IV shows correlation of ultrasonogram and Cystoscopy of the urinary bladder. These two, one noninvasive and the other an invasive technique respectively, are the mainstay of investigative procedure in the diagnosis of mass within the urinary bladder. Urine cytology particularly voided urine cytology is also a non-invasive technique having a high diagnostic yield, especially in high-grade lesion of urinary bladder. 
Out of 57 cases, ultrasonogram gave positive result in 54 cases ( $95 \%$ approximately), whereas out of 55 cases, cystoscopy gave positive result in 54 cases (98\% approximately). Two cases were excluded since the mass was located within the renal pelvis and cystoscopy was not done in these cases.

Table IV: Ultrasonogram and cystoscopic finding correlation.

\begin{tabular}{llllll}
\hline Investigation & Total No. & Positive & Percentage & Negative & Percentage \\
\hline Ultrasonogram & 57 & 54 & $94.74 \%$ & 03 & $5.26 \%$ \\
Cystoscopy & $55^{*}$ & 54 & $98.19 \%$ & 01 & $1.81 \%$ \\
\hline
\end{tabular}

* Two mass in renal pelvis: Cystoscopy was not done.

Histologic diagnosis of 57 cases:

Table V shows the histologic diagnoses of 57 cases. There were 14 (24.56\%) non-invasive papillary tumors, 1 (1.76\%) carcinoma in-situ and 42 (73.68\%) invasive carcinomas of all grades and types.

Table V: Histologic diagnosis of 57 cases.

\begin{tabular}{|c|c|c|c|}
\hline No. & Histologic Diagnosis & No. of cases & Percentage \\
\hline 1. & Non-invasive Papillary tumor & 14 & $24.56 \%$ \\
\hline 2. & Non-papillary carcinoma in situ & 01 & $1.76 \%$ \\
\hline \multirow[t]{2}{*}{3.} & Invasive carcinoma (all grades/types) & 42 & $73.68 \%$ \\
\hline & Total & 57 & $100 \%$ \\
\hline
\end{tabular}

\section{Discussion:}

The aim of this study was to establish diagnostic usefulness of voided urine cytology and ultrasonography and cystoscopy in the evaluation of urinary bladder tumor.

Cytologic study of the urine as a diagnostic procedure was first introduced by Papanicolaou and Marshall in $1945 .{ }^{21}$ Urinary tract cytology is requested by Urologists as a diagnostic aid rather than as a detection technique for urinary tract cancer. The Urologists seek the assistance of diagnostic cytology only in those cases where a suggestion of a malignant neoplasm of the urinary tract exists. $^{22}$

The practical value of cytological examination of urine is, first in reducing the chance of biopsy sampling error and second, in the detection of cancer in patients who refuse frequent cystoscopy. ${ }^{23}$ Urine cytology before review cystoscopy may help in avoiding unnecessary general anaesthesia and reduce both cost and patient morbidity. ${ }^{16}$ As a follow up procedure, cytology may enhance the value as well as reduce the number of cystoscopy performed by accurately indicating when a search for cancer needs to be undertaken. ${ }^{12}$

Since $50-70 \%$ of the patients with transitional cell carcinoma particularly the superficial transitional cell carcinoma will experience a recurrent tumor, it is important to have a non- invasive assay that can predict the likelihood of bladder transitional cell carcinoma recurrence. ${ }^{24}$

This study demonstrated the accuracy of cytology by evaluating comparison of cytologic and histopathologic diagnoses. In the present study, results of urine cytology of 57 patients were correlated with tissue diagnosis. Histopathological diagnoses were non-invasive papillary tumors 14 (25\%), carcinoma in-situ 1 (2\%) and invasive carcinoma $42(74 \%)$.

Out of 57 cases, cytological diagnosis was positive in 53 cases (93\%) and negative in 4 cases (7\%). There were no atypical or suspicious cases. Of the total cases, cytology was negative only in $7 \%$ cases. Among 42 invasive carcinomas 14 (33\%) grade III invasive flat carcinoma, one (2\%) invasive papillary adenocarcinoma and two (5\%) 
invasive squamous cell carcinoma, each of them gave $100 \%$ positive cytology. Of the remaining $25(60 \%)$ invasive papillary TCC, 21 were in grade II and 4 were in grade III. They gave $90 \%$ and $100 \%$ positive cytologic results.

Ultrasound is a very useful modality in diagnosis of urinary bladder neoplasm, which is cheap, easily available, noninvasive and with negligible discomfort. So, sonographic scan is recommended as a useful diagnostic modality in respect of urinary bladder neoplasm

Ultrasonogram of urinary bladder gave 95\% (approx.) positive results with a comment of mass in the urinary bladder and cystoscopy detected tumor mass in $98 \%$ cases. In one case (2\%) it failed to detect any abnormality. Out of 54 positive cystoscopy cases, 38 were papillary and 16 were sessile. The positive cytological correlation was $90 \%$ and $100 \%$ respectively.

In a study, gross or microscopic haematuria was the most consistent sign noted in $82-94 \%$ of the case. $^{12}$ So one must evaluate haematuria cases with great caution and along with other invasive and non invasive techniques, urine cytology and ultrasonography offers one of the mainstay as a diagnostic adjunct. Out of 57 cases, 3 cases failed to give positive results in Ultrasonogram (USG) of the urinary tract. USG gave false positive result when there is blood clot or necrotic tissue within the bladder. False negative results are due to carcinoma in-situ and very small sessile or papillary lesions.

Cystoscopy gave false negative result in $2 \%$ cases. This is due to carcinoma in-situ of bladder. Carcinoma in-situ presents as red congested focal or diffuse oedematous area resembling “cobblestone epithelium”, "velvety redness" or interstitial cystitis and in some cases, there might be no Cystoscopic abnormalities whatever. ${ }^{6}$ Only an experienced cystoscopist can identify carcinoma in-situ with great caution. Sessile tumors are mainly invasive and they gave more cytologically positive results than that of papillary tumors. ${ }^{25}$

Fifty-two tumors had a diameter ranging 1.1- 6 $\mathrm{cm}$. Single lesions were seen in 40 cases, double lesion in one case and multiple in 13 cases. Ro and co-workers mentioned that the number of papillary tumors are an important risk factor for recurrence but is not a significant determinant of invasive disease. Cutler and associates showed that patients with a single tumor has a $67 \%$ chance of recurrence, in contrast to a $90 \%$ chance if their growth were initially multiple. The disease free interval for patients with multiple tumors also was shorter. Dalesia and co-workers found the number of tumors to be the single most important factor influencing the likelihood of development of new tumors. Lerman and associates also demonstrated significantly different recurrence rates for single lesion (31\%) versus multiple lesions (66\%). Furthermore the probability of later invasive carcinomas nearly tripled in-patients with multiple lesions on presentation (13.6\%) compared with those who presented with a single lesion $(4.6 \%){ }^{5}$

Consistent with previously published data, this study showed the highest diagnostic accuracy with high-grade tumors and lowest with low-grade tumors with maximum invasion limited to the lamina propria. The percentage of false negative reports in this series can be partly due to poor sampling or low-grade tumor or due to poor techniques adopted. In a study of 91 patients, urine cytology was $75 \%$ sensitive and $94 \%$ specific. $^{26}$ Sensitivity of urine cytology in 860 patients with urinary tract cancer was $77 \%$ and the specificity is $97 \%{ }^{19}$ L.G.Koss in a study of 183 cases having bladder cancer, described the sensitivity of $82.5 \%$ for all tumors. If one excludes grade-I and II tumors, the sensitivity for all high-grade lesions of $100 \%$ for flat CIS. ${ }^{6,9}$ In low-grade cancers, the sensitivity is $66.2 \%$, for grade-I $16.7 \%$ and for grade-II $71.6 \%$. The specificity was $100 \%$ since there are no false positive cases. ${ }^{9}$ In this study the non- invasive technique was adopted and voided urine specimen \& USG was chosen. The purpose of this is to see how a simple test can be helpful in diagnosing malignant tumors of urinary bladder.

Urine cytology is not a popular method for screening or detection of malignancy in our country. Urine cytology has immense value as a non- invasive technique, not only as a diagnostic tool but also as a prognostic factor. Positive 
cytology correlates well with grade, stage and prognosis of bladder tumors, so it can be used in the detection, diagnosis and follow-up of the primary tumor and for recurrent tumors receiving therapy. Even after cystoscopy and transurethral resection of bladder tumor (TURBT), urine cytology play important role to assess the prognosis of the tumor. If urine cytology shows high-grade cancer cells and tissue diagnosis after removal, reveals a low-grade tumor, then the conclusion will be that besides the low-grade cancer, there might be high-grade abnormality within the bladder not detected during cystoscopic removal. So the outcome of patients will differ in such cases. A tumor localized within a diverticulam or higher up in the tract, may escape detection during cystoscopy but urine cytology can give a meaningful diagnosis in such cases, if the tumor is of high grade. This possibility should be kept in mind and a thorough search should be made to detect the lesions.

As urine cytology have the advantage of sampling whole bladder mucosa, invasive diseases or flat CIS can be diagnosed long before identification by USG or cystoscopy, thus helps in increasing survival rate of the patients.

\section{Conclusion:}

A more or less definitive pre-operative diagnosis of malignant tumors of urinary bladder can help the Urologists for making decision about the plan of surgery or of radiotherapy or chemotherapy. Among all the methods of making a preoperative diagnosis of urothelial cancers, urine cytology and USG are important non invasive methods which can give results very closer to the histologic diagnosis or at least can say about the nature of the tumor. Urothelial cancer presents with haematuria. To evaluate every cases, non invasive technique like voided urine cytology and USG could be done as a first line cost effective method in the detection, diagnosis and follow up of primary and recurrent urothelial cancers where facilities for other investigations are limited.

\section{References}

1. Reuter VE. The Lower Urinary Tract. In: Sternberg SS. Ed: Diagnostic Surgical Pathology. New York. Raven press. 1989:pp1355-1392.

2. Rosai J. Ackerman's Surgical Pathology. $7^{\text {th }}$ edition. St. Louis. C.V. Mosby. 1989: pp 898-922.

3. Kern WH. Urinary Tract. In: Bibbo M. Ed: Comprehensive Cytopathology. Philadelphia. W.B. Saunders company. 1991:pp433-467.

4. Cotran RS, Kumar V, Robbins SL. Pathologic Basis of Disease. $5^{\text {th }}$ Edition. Philadelphia. W.B. Saunders company. 1994:pp 991-1005.

5. Ro JY, Staerkel GA, Ayala AG. Cytologic and histologic features of superficial bladder cancer. Urologic Clinics of North America 1992; 19: 435453.

6. Koss LG. Diagnostic Cytology and its Histopathologic Bases. $4^{\text {th }}$ Edition. Philadelphia. JB Lippincott. 1992: pp 935-986.

7. Koss LG, Tiamson EM, Robbins MA. Mapping cancerous and precancerous bladder changes. JAMA 1974; 227: 281-286.

8. Kern $\mathrm{WH}$. The grade and pathologic stage of bladder cancer. Cancer 1984; 53: 1145-1189.

9. Koss LG, Deitch D, Ramanathan R, Sherman AB. Diagnostic value of cytology of voided urine. Acta Cytologica 1985; 29: 810- 816.

10. Badalament RA, Hermansen DK, Kimmel M, Gay $\mathrm{H}$, Herr HW, Fair WR, Whitmore jr. WF, Melamed MR. The sensitivity of bladder wash flow cytometry, bladder wash cytology and voided cytology in the detection of bladder carcinoma. Cancer 1987; 60: 1423-1427.

11. Ashley DJB. Evan's Histological Appearances of Tumours. $4^{\text {th }}$ edition. London. Churchill Livingstone. 1990; pp 992-949.

12. Geisse LJ, Tweeddate DN. Pre-clinical cytological diagnosis of bladder cancer. The Journal of Urology 1974; 120: 51-56.

13. Farrow GM. Pathologist's role in bladder cancer. Seminars in Oncology 1979; 6: 194-206.

14. Papanicolaou GN, Marshall VF. Urine sediment smears as a diagnostic procedure in cancers of the urinary tract. Science 1945: 101: 519-520.

15. Foot NC, Papanicolaou GN, Holmquist ND, Seybolt JF. Exfoliative cytology of urinary sediments. Cancer 1958; 11: 127-137.

16. Toivonen $\mathrm{T}$, Hasto $\mathrm{AL}$. Large volume cytocentrifuge for processing alcohol fixed 
cytologic specimens. Acta Cytologica 1991; 35: 269-272.

17. Murphy WM, Soloway MS, Jukkola AF, Crabtree WM, Ford KS. Urinary cytology and bladder cancer. Cancer 1984; 54: 1555-1565.

18. Wiener HG, Vooijs GP, Grootenboer BVH, Accuracy of urinary cytology in the diagnosis of primary and recurrent bladder cancer. Acta Cytologica 1993; 37: 163- 169.

19. Kern WH. The diagnostic accuracy of sputum and urine cytology. Acta Cytologica 1988; 32: 651-654.

20. Loh CS, Spedding AV, Ashworth MT, Kenyon WE, Desmond $A D$. The value of exfoliative urine cytology in combination with flexible cystoscopy in the diagnosis of recurrent transitional cell carcinoma of the urinary bladder. British Journal of Urology 1996; 77: 655-658.
21. Schooness R, Gamarra MG, Moore RH, Murphy GP. The diagnostic value of urinary cytology inpatients with bladder carcinoma. The Journal of Urology 1971; 106: 693-696.

22. Holmquist ND. Detection of urinary tract cancer in urinalysis specimens in an out patient population. A.J.C.P. 1988; 89: 499-504.

23. Lerman RI, Hutter RVP, Whitmore Jr. WF. Papilloma of the urinary bladder. Cancer 1970; 25: 333-342.

24. Giella JG, Ring K, Olsoon CA, Karp FS, Benson $M C$. The predictive value of flow cytometry and urinary cytology in the follow up of patients with TCC of the bladder. The Journal of Urology 1992; 148: 293-296.

25. Jordan AM, Weingarten J, Murphy WM. Transitional cell neoplasms of the urinary bladder. Cancer 1987; 60: 2726-2774.
All correspondence to Dr. Shah Md. Badruddoza Professor, Department of Pathology, Rajshahi Medical College Rajshahi Email: smbdbd22@gmail.com 